\title{
ANÁLISE CRÍTICA DA SERIOGRAFIA DO ESÔFAGO, ESTÔMAGO E DUODENO EM UM SERVIÇO DE RADIOLOGIA DE UM HOSPITAL GERAL*
}

\author{
Marcelo Souto Nacif ${ }^{1}$, Gustavo Federico Jauregui ${ }^{2}$, Viviane Brady Rocha ${ }^{2}$, Adjane Barcia ${ }^{2}$, \\ Ana Paula Boechat ${ }^{2}$, Alderico Mendonça Neto ${ }^{2}$, Silvio Silva Neto ${ }^{2}$, Ernesto Misael Cintra Osterne ${ }^{3}$, \\ Ricardo Andrade Fernandes de Mello ${ }^{4}$, Alair Augusto Sarmet Moreira Damas dos Santos ${ }^{5}$
}

Resumo OBJETIVO: Foram revisados os exames de seriografia do esôfago, estômago e duodeno (SEED), a fim de demonstrar os principais diagnósticos e achados radiológicos, o atual benefício para o paciente e a viabilidade financeira para os serviços de radiologia. MATERIAIS E MÉTODOS: Estudo retrospectivo e descritivo realizado a partir do levantamento dos exames realizados no período de 6/3/2001 a 12/4/2002 no Serviço de Radiologia do Hospital Santa Cruz da Beneficência Portuguesa de Niterói. RESULTADOS: Do total de 270 exames, $115(42,5 \%)$ apresentavam alterações radiológicas (anatômicas, congênitas, adquiridas, funcionais, pépticas ou relacionadas a cirurgias) e 155 (57,5\%) exames foram normais. Em relação à faixa etária, $197(72,9 \%)$ eram pacientes de zero a 12 anos incompletos e $73(27,1 \%)$ tinham mais de 12 anos. CONCLUSÃO: A SEED não deve cair em desuso e sim se manter como um importante método complementar para o estudo das doenças do trato gastrintestinal superior, principalmente em crianças e nos casos cirúrgicos, pelo seu indubitável benefício aos pacientes. Assim, devemos ter em mente que o exame contrastado e o exame endoscópico do trato gastrintestinal superior são complementares e não excludentes, quando consideramos as vantagens e as desvantagens de cada método. A SEED é um exame simples, rápido, relativamente barato e com poucos riscos para os pacientes.

Unitermos: Alterações do trato gastrintestinal alto; Estudo contrastado; Diagnósticos diferenciais.

Abstract Critical analysis of upper gastrointestinal contrast examinations at a radiology service in a general hospital. OBJECTIVE: To review upper gastrointestinal tract (UGI) contrast examinations in order to establish the main diagnoses and radiological findings, the actual benefits to the patients and the costs for the department of radiology. MATERIALS AND METHODS: This is a retrospective study of $270 \mathrm{UGI}$ contrast examinations performed between 6/3/2001 and 12/4/2002 at the Department of Radiology of "Hospital Santa Cruz/ Beneficência Portuguesa de Niterói", Niterói, RJ, Brazil. RESULTS: A total of 270 examinations were reviewed. Radiological findings (anatomical, congenital, acquired, peptic or surgery related findings) were seen in 115 $(42.5 \%)$ examinations while $155(57.5 \%)$ examinations were normal. The age range was 0-12 years in 197 $(72.9 \%)$ patients whereas $73(27.1 \%)$ patients were above 12 year-old. CONCLUSION: Due to the clear benefit to the patients, UGI contrast examinations should continue to be used as an important complementary method to study UGI diseases, particularly in children and surgical cases. Therefore, we should keep in mind that UGI contrast examinations and endoscopy are complementary methods of investigation when comparing its advantages and disadvantages. UGI contrast examination is a fast, simple, and relatively low cost procedure. In addition, it presents low risk to the patients.

Key words: Upper gastrointestinal tract diseases; Contrast examination; Differential diagnosis.

* Trabalho realizado no Instituto de Pós-Graduação Médica Carlos Chagas (IPGMCC), Hospital Santa Cruz/Beneficência Por tuguesa de Niterói, Niterói, RJ, e na Faculdade de Medicina de Teresópolis, Centro de Ciências Biomédicas da Fundação Educacional Serra dos Órgãos (FMT-CCBM - FESO), Teresópolis, RJ.

1. Professor Auxiliar da Disciplina de Radiologia da FMT-CCBM - FESO, Pós-graduando do Curso de Especialização em Radiologia do IPGMCC, Mestrando em Radiologia pela Universidade Federal do Rio de Janeiro (UFRJ).

2. Médicos Pós-graduandos do Curso de Especialização em Radiologia do IPGMCC.

3. Monitor da Disciplina de Radiologia da FMT-CCBM - FESO. 4. Especialização em Radiologia pelo IPGMCC, Membro Titular do Colégio Brasileiro de Radiologia e Diagnóstico por Imagem (CBR), Mestre em Radiologia pela UFRJ.

5. Professor Titular do Curso de Especialização em Radiologia do IPGMCC, Mestre e Doutor em Radiologia pela UFRJ, Chefe do Serviço de Imagem do Hospital de Clínicas de Niterói e da VOT Imagem, Professor Adjunto e Chefe do Serviço de Radiologia do HUAP-UFF.

\section{INTRODUÇÃO}

Desde a descoberta da utilização da radiação X para o diagnóstico em medicina, em 1896, o uso de substâncias como o bismuto, o óxido de ferro e os sais de chumbo já estava experimentalmente sendo posto em prática no estudo do trato gastrintestinal superior (TGIS). Em 1904, Hermann

Endereço para correspondência: Dr. Marcelo Souto Nacif. Rua Álvares de Azevedo,130/704, Icaraí. Niterói, RJ, 24220-021. E-mail:marcelonacif30@hotmail.com/msnacif@yahoo.com.br Recebido para publicação em 27/5/2003. Aceito, após revisão, em 26/11/2003.
Rieder, na Alemanha, introduziu a "papa de bismuto", que era tóxica e não muito apetitosa, mas que passou a ser muito estudada nos serviços da Europa e dos Estados Unidos, o que trouxe muitos dados sobre os aspectos das doenças e achados da normalidade do TGIS. Já em 1910, C. Bachem e H. Günther introduziram o sulfato de bário, meio de contraste mais barato e que pode ser industrializado. Quando se somou o ar, realizando-se a técnica do duplo meio de contraste, isto fez com que os radiologistas aperfeiçoassem as técnicas, posições e os métodos de estudo, 
contribuindo enormemente para o diagnóstico até de pequenas alterações da mucosa e estágios iniciais de cânceres, o que, por um longo tempo, ajudou e muito os clínicos e cirurgiões no manejo de seus pacientes no mundo inteiro ${ }^{(1-3)}$

O avanço tecnológico na medicina, com o advento de fármacos mais eficazes, utilização de terapêuticas empíricas, pHmetria, manometria, associada à endoscopia digestiva alta, fez com que o exame contrastado tivesse um declínio gradual e contínuo, como é evidenciado atualmente. Mas é importante que os clínicos tenham ciência que o exame endoscópico é caro, invasivo e com morbidade significativa relacionada ao uso de sedação e ao risco de perfuração, principalmente em mãos inexperientes. Dessa forma, a possibilidade de uma análise dinâmica ou estática através do estudo do ato da deglutição, observação das contrações esofágicas, do esvaziamento gástrico e do peristaltismo das alças, entre outros, são aspectos que nenhum método mostra com tanta clareza como o estudo contrastado. Portanto, o estudo contrastado não deve cair em desuso e sim ser um método complementar, barato, rápido, simples, sem riscos para o paciente, e que deve continuar a ser um exame importante para o estudo das doenças do TGIS ${ }^{(4)}$.

Em nosso meio, até onde foi possível pesquisar, não foram realizados estudos mostrando como está a utilização do exame contrastado do TGIS nos serviços de radiologia, para reavaliar sua grande importância no diagnóstico e orientação médica. Conforme exposto, não podemos deixar de realizar estudos sobre o assunto para que sempre consigamos uma melhora na qualidade dos exames e no atendimento dos pacientes nos serviços de radiologia.

\section{Objetivo}

Descrever e analisar os principais diagnósticos e achados radiológicos, o número de exames normais e com alterações radiológicas nas seriografias do esôfago, estômago e duodeno (SEED) realizadas no período de 6/3/2001 a 12/4/2002, a fim de fornecer informações sobre o atual benefício para o paciente e sobre o custo-benefício para o serviço de radiologia que continua a realizar este tipo de exame. Propusemo-nos a descrever, também, as caracte- rísticas dos pacientes atendidos no nosso serviço de radiologia quanto a sexo, faixa etária e as regiões de moradia de origem. Assim, esperamos contribuir para a melhoria da assistência nos serviços de radiologia da nossa região.

\section{MATERIAIS E MÉTODOS}

Este estudo observacional, descritivo e retrospectivo, foi realizado no Serviço de Radiologia do Hospital da Beneficência Portuguesa de Niterói (Santa Cruz Scan), serviço onde funciona o Curso de Especialização em Radiologia do Instituto de PósGraduação Médica Carlos Chagas (IPGMCC), e que está situado na cidade de Niterói, RJ. O trabalho foi realizado a partir de levantamento dos laudos e pedidos médicos armazenados no banco de dados dos computadores do Santa Cruz Scan. Foram selecionadas todas as SEED realizadas no período compreendido entre 6/3/2001 e $12 / 4 / 2002$, num total de 270 exames, o que veio a ser a nossa amostra.

\section{RESULTADOS}

Do total de 270 exames realizados no período citado, 115 (42,5\%) apresentavam alterações radiológicas e 155 (57,5\%) exames foram normais.

Observamos que, em relação ao sexo, 133 pacientes $(49,2 \%)$ eram do sexo masculino e $137(50,8 \%)$ eram do sexo feminino. Quanto à idade, o paciente mais novo tinha 11 dias de vida e o mais velho, 87 anos, sendo a idade média de 43,5 anos. Quanto à faixa etária, 197 (72,9\%) eram pacientes de zero a 12 anos incompletos e $73(27,1 \%)$ pacientes tinham mais de 12 anos. Os dados relativos à faixa etária estão demonstrados na Tabela 1.
Quanto à região de moradia, a cidade de São Gonçalo, com 121 pacientes (44,8\%), foi a de maior predomínio. A Tabela 2 mostra a origem dos pacientes quanto às diferentes cidades da região.

Tabela 2 Região de origem (moradia) dos pacientes.

\begin{tabular}{|l|c|c|}
\hline \multicolumn{1}{|c|}{ Região } & № de pacientes & Porcentagem \\
\hline São Gonçalo & 121 & $44,8 \%$ \\
Niterói & 102 & $37,8 \%$ \\
Itaboraí & 15 & $5,6 \%$ \\
Rio de Janeiro & 8 & $3,0 \%$ \\
Saquarema & 6 & $2,2 \%$ \\
Maricá & 4 & $1,5 \%$ \\
Tanguá & 3 & $1,1 \%$ \\
Outras & 11 & $4,1 \%$ \\
\hline
\end{tabular}

Do total de 161 diagnósticos e achados radiológicos, podemos observar que 67 $(41,6 \%)$ dos achados foram por refluxo gastroesofágico. A hérnia de hiato por deslizamento foi evidenciada em 24 (14,9\%), o trânsito esofagiano alentecido em 11 $(6,8 \%)$, e ondas terciárias também em 11 $(6,8 \%)$ casos. Os demais achados estão apresentados na Tabela 3. Exemplos de diagnósticos e achados radiológicos são mostrados nas Figuras de 1 a 6 .

$\mathrm{Na}$ avaliação do custo-benefício da realização deste exame, observamos que no período estudado obtiveram-se, em média, nos exames de crianças, $\mathrm{R} \$ 16,12$ de gasto direto nos exames realizados, e o pagamento foi, em média, de $\mathrm{R} \$ 65,00$ para os exames realizados; portanto, o lucro no período estudado foi, em média, de $\mathrm{R} \$ 48,88$, sem contar os insumos. Nos exames de adultos, obtiveram-se R $\$ 19,76$ de gasto direto nos exames realizados, e o pagamento foi, em média, de $\mathrm{R} \$ 83,00$ para os exames realizados, sendo o lucro no período

Tabela 1 Faixa etária dos pacientes.

\begin{tabular}{|ll|r|r|}
\hline \multicolumn{2}{|c|}{ Faixa etária } & № de pacientes & Porcentagem \\
\hline - Recém-nato & (0-28 dias) & 4 & $1,5 \%$ \\
- Lactente & (29 dias-2 anos incompletos) & 130 & $48,1 \%$ \\
- Pré-escolar & (2 anos-6 anos incompletos) & 42 & $15,7 \%$ \\
- Escolar & (6 anos-12 anos incompletos) & 21 & $7,8 \%$ \\
- Adolescente & (12 anos-18 anos incompletos) & 2 & $0,7 \%$ \\
- Adulto jovem & (18 anos-35 anos) & 7 & $2,6 \%$ \\
- Adulto & (35 anos-65 anos) & 31 & $11,5 \%$ \\
- Idoso & (mais de 65 anos) & 33 & $12,2 \%$ \\
\hline
\end{tabular}


Tabela 3 Diagnósticos e achados radiológicos.

\begin{tabular}{|l|c|c|}
\hline & № de pacientes & Porcentagem \\
\hline Refluxo gastroesofágico grau III & 25 & $15,5 \%$ \\
Hérnia de hiato por deslizamento & 24 & $14,9 \%$ \\
Refluxo gastroesofágico grau II & 22 & $13,7 \%$ \\
Refluxo gastroesofágico grau I & 14 & $8,7 \%$ \\
Trânsito esofagiano alentecido & 11 & $6,8 \%$ \\
Ondas terciárias & 11 & $6,8 \%$ \\
Refluxo gastroesofágico grau IV & 6 & $3,7 \%$ \\
Gastrectomia total & 6 & $3,7 \%$ \\
Divertículo duodenal & 5 & $3,1 \%$ \\
Megaesôfago-acalasia & 4 & $2,5 \%$ \\
Fundoplicatura & 4 & $2,5 \%$ \\
Gastrite & 4 & $2,5 \%$ \\
Fístula & 3 & $1,9 \%$ \\
Retardo do esvaziamento gástrico & 3 & $1,9 \%$ \\
Úlcera gástrica & 3 & $1,9 \%$ \\
Divertículos esofagianos de tração & 2 & $1,2 \%$ \\
Divertículo de Zenker & 2 & $1,2 \%$ \\
Hérnia de hiato paraesofagiana & 2 & $1,2 \%$ \\
Gastrectomia parcial & 2 & $1,2 \%$ \\
Úlcera esofagiana & 2 & $1,2 \%$ \\
Alargamento do arco duodenal & 1 & $0,6 \%$ \\
Diafragma antral & 1 & $0,6 \%$ \\
Divertículo no fundo gástrico & 1 & $0,6 \%$ \\
Retenção de contraste nas valéculas & 1 & $0,6 \%$ \\
Úlcera duodenal & $0,6 \%$ \\
Volvo gástrico & $0,6 \%$ \\
\hline
\end{tabular}

estudado, em média, de R\$ 63,24. Dessa forma, observou-se que a porcentagem de lucro nos exames de adultos foi de $46 \%$ do pagamento e nos de criança, de $47 \%$. Observar os resultados na Tabela 4.

\section{DISCUSSÃO}

Atualmente, observando o grande declínio no uso da SEED e até do interesse dos radiologistas em aprender a realizar e interpretar os exames contrastados, optamos por mostrar o real benefício deste exame e a importância deste procedimento diagnóstico dentro de um hospital geral. A SEED pode ser utilizada de acordo com a experiência de cada serviço, mas também pode servir como procedimento de acompanhamento, que muitas vezes é conclusivo, e quando comparado com a melhora clínica do paciente pode-se não necessitar da utilização de outros exames ${ }^{(5-11)}$.

É sabido que a endoscopia é o exame de escolha para as doenças do TGIS, já que

Tabela 4 Relação custo-benefício do método. era a pneumonia de repetição. Estes muitas vezes possuíam o diagnóstico cintilográfico de refluxo e estavam realizando a SEED para observar a coexistência de alterações funcionais ou morfológicas que estivessem levando à doença ${ }^{(7,8)}$. Maglinte et al. ${ }^{(16)}$ estudaram 500 exames contrastados do esôfago, estômago e duodeno e observaram, em $15 \%$ dos exames, achados referentes a doenças do esôfago. Na nossa casuística este valor foi de $54,5 \%$ e se deu, novamente, pela faixa etária dos pacientes do nosso estudo.

O exame contrastado do TGIS ainda é um exame com grande valia para o estudo pré e pós-operatório, visualização de anastomoses, fístulas e demonstração da condição cirúrgica do segmento submetido ao procedimento no pós-operatório. A avaliação pré e pós-operatória talvez seja a grande indicação deste método atualmente $\mathrm{e}^{(\mathbf{5 , 6 , 1 7})}$.

Observamos que muitas clínicas de radiologia estão deixando de realizar o exame contrastado. Muitos alegam que o convênio não paga, outros que não se tem benefício com a realização do exame, e na verdade as desculpas são inúmeras. É certo que, atualmente, com os outros métodos de diagnóstico, a radiologia convencional passou a ficar em segundo plano e os lucros obtidos com outros exames sem sombra de dúvida são maiores. Porém, estamos exercendo a arte da radiologia e precisamos, acima de tudo, nos preocupar com o paciente e com o real benefício que podemos proporcionar ao realizar cada exame. Por isso optamos por demonstrar, como visto na Tabela 4, que ainda podemos contribuir e muito com o diagnóstico e orientação, além de conseguirmos um lucro relativo importante.

Toda a consideração em relação ao lucro deve ser avaliada de maneira mais profunda. É óbvio que o preço destes exames é menor do que de uma ressonância magnética ou de uma tomografia computadorizada, por exemplo. Entretanto, se consi-

\begin{tabular}{|l|c|c|c|c|}
\hline & № de exames & $\begin{array}{c}\text { Média de gasto } \\
\text { por exame }\end{array}$ & $\begin{array}{c}\text { Média de pagamento } \\
\text { por exame }\end{array}$ & Lucro \\
\hline Crianças* $^{*}$ & 197 & $\mathrm{R} \$ 16,12$ & $\mathrm{R} \$ 65,00$ & $\mathrm{R} \$ 48,88$ \\
Adultos $^{\dagger}$ & 73 & $\mathrm{R} \$ 19,76$ & $\mathrm{R} \$ 83,00$ & $\mathrm{R} \$ 63,24$ \\
\hline
\end{tabular}

* Nas crianças considerou-se a utilização de seis filmes $(18 \times 24)$, um copo de bário e uma seringa. ${ }^{\dagger}$ Nos adultos considerou-se a utilização de seis filmes $(24 \times 30)$, três filmes $(18 \times 24)$ e um copo de bário. 


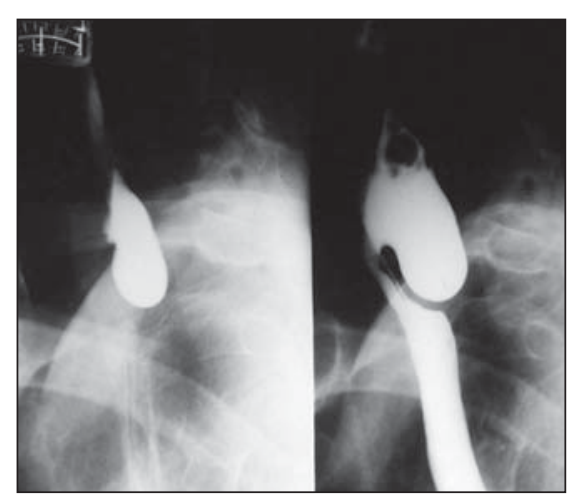

Figura 1. Divertículo de Zenker.

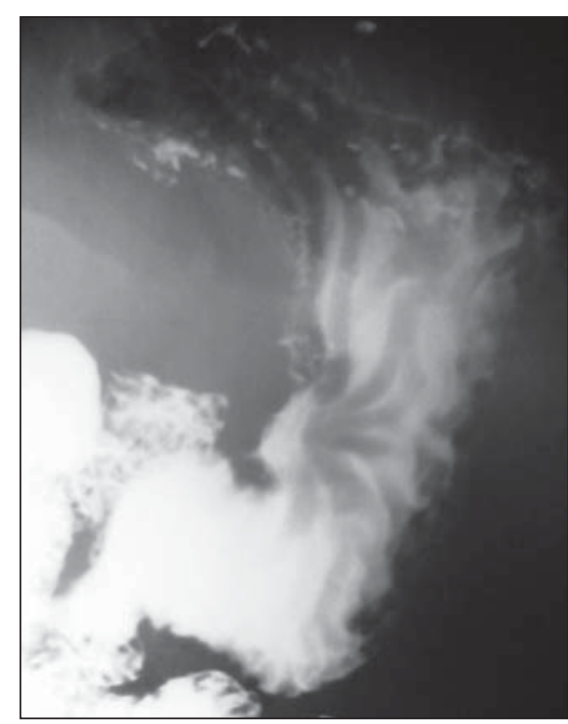

Figura 3. Úlcera gástrica maligna.

derarmos os valores recebidos, a pouca depreciação relativa dos equipamentos de radiologia convencional em comparação com os de ressonância magnética e tomografia computadorizada, e se tivermos um serviço que tenha direcionamento destes exames numa determinada cidade ou região, associando-se com a otimização do tempo e da técnica radiológica, com absoluta certeza poderíamos garantir que estes exames justificariam seu custo. Outro dado importante é que estamos num momento,

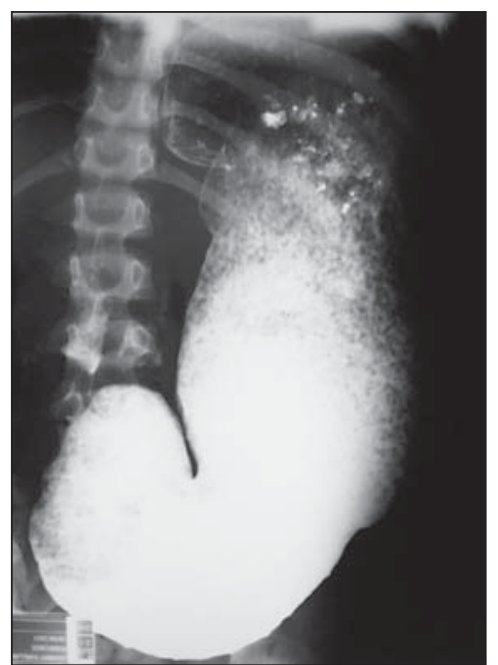

A

Figura 2. Diafragma antral no pré (A) e pós-operatório (B), respectivamente.

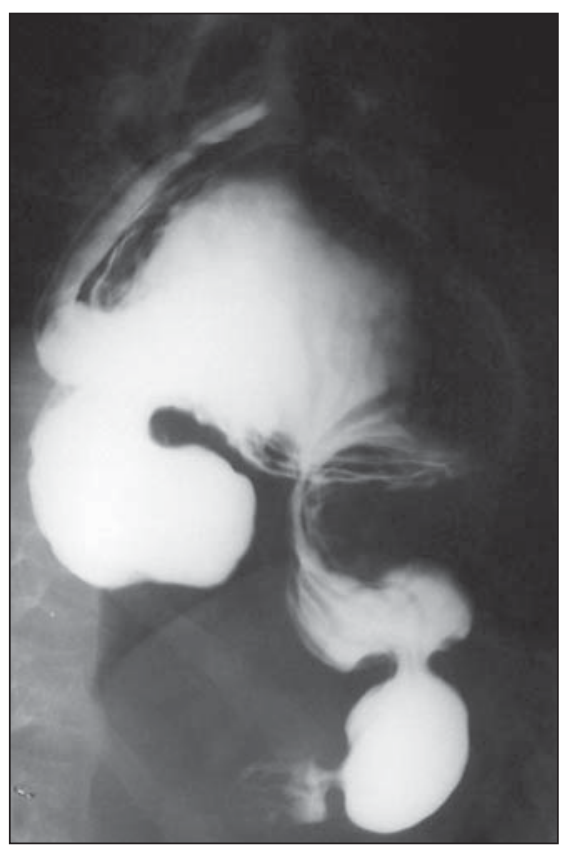

Figura 4. Volumosa hérnia diafragmática paraesofagiana.

no país, de revalorização da radiologia convencional, e precisamos implementar o ensino e a execução deste tipo de exame.

\section{CONCLUSÃO}

A SEED já é realizada há quase um século, tendo inegável importância no estudo das doenças e da anatomia do TGIS, além de ser um exame barato, rápido, simples e quase sem riscos para o paciente. A SEED não deve cair em desuso, principal-

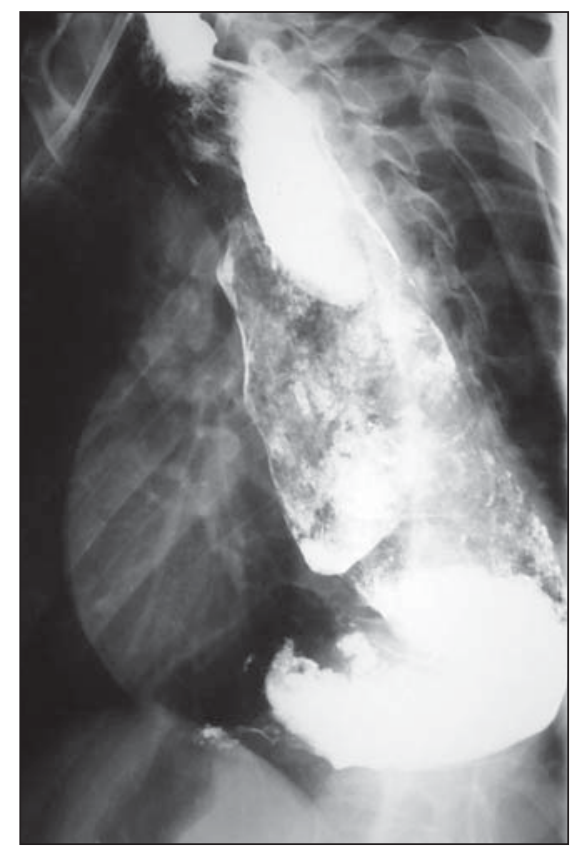

Figura 5. Megaesôfago.

mente nos casos cirúrgicos (pré e pós-operatório), pelo seu real benefício aos pacientes. Dessa forma, temos que ter em mente que a SEED e a endoscopia são exames complementares e nunca excludentes, considerando-se as limitações e as vantagens de cada método. A SEED é um exame que, mesmo com os insumos, ainda continua a dar lucro para o serviço de radiologia. Em vista do que apresentamos, esperamos ter contribuído para a melhor assistência nos serviços de radiologia. 


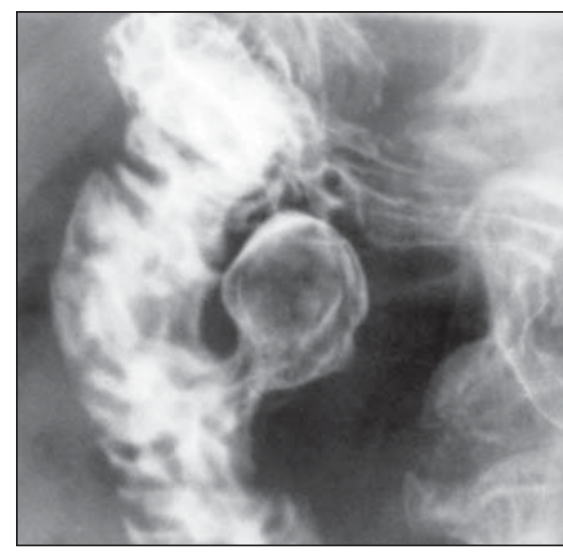

Figura 6. Divertículo duodenal.

\section{REFERÊNCIAS}

1. Ottenjann R, Elster K. Expanding the power of the senses. In: Ottenjann R, Elster K, Cohen S, et al., eds. Atlas of diseases of the upper gastrointestinal tract. Philadelphia, PA: Smith Kline Corporation, 1980:26-7.

2. Sato E, Rosa ACF, Ogasawara AM, Costacurta MA, Cerri GG. Exames contrastados do trato digestivo alto. Radiol Bras 1999;32:263-72.

3. Freitas LO, Nacif MS. Radiologia prática para o estudante de medicina. Rio de Janeiro, RJ: Revinter, 2001.

4. Op den Orth JO. Use of barium in evaluation of disorders of the upper gastrointestinal tract: current status. Radiology 1989;173:601-8.

5. Laufer I. Esophagus. In: Laufer I, ed. Double contrast gastrointestinal radiology with endoscopic correlation. Philadelphia, PA: Saunders, 1979:79_ 128.

6. Laufer I. Stomach. In: Laufer I, ed. Double contrast gastrointestinal radiology with endoscopic correlation. Philadelphia, PA: Saunders, 1979:155-240.

7. Stevenson GW, Laufer I. Duodenum. In: Laufer I, ed. Double contrast gastrointestinal radiology with endoscopic correlation. Philadelphia, PA: Saunders, 1979:331-72.

8. Meyer JII. Motility of the stomach and gastroduodenal junction. In: Johnson LR, ed. Physiology of the gastrointestinal tract. 2nd ed. New York, NY: Raven Press, 1987:613-30.

9. Thoeni RF, Goldberg HI, Ominsky S, Cello JP. Detection of gastritis by single- and double-contrast radiography. Radiology 1983;148:621-6.

10. de Oliveira JM, Birgisson S, Doinoff C, et al. Timed barium swallow: a simple technique for evaluating esophageal emptying in patients with achalasia. AJR 1997;169:473-9.
11. Creteur V, Laufer I, Kressel HY, et al. Drug-induced esophagitis detected by double-contrast radiography. Radiology 1983;147:365-8.

12. Thoeni RF, Cello JP. A critical look at the accuracy of endoscopy and double-contrast radiography of the upper gastrointestinal (UGI) tract in patients with substantial UGI hemorrhage. Radiology 1980;135:305-8.

13. Laufer I. A simple method for routine double-contrast study of the upper gastrointestinal tract. Radiology 1975;117:513-8.

14. Laufer I. Barium studies of the upper gastrointestinal tract. In: Gore RM, Levine MS, eds. Textbook of gastrointestinal radiology. 2nd ed. Philadelphia, PA: Saunders, 2000:272-84.

15. Levine MS. Esophagus: differential diagnosis. In Gore RM, Levine MS, eds. Textbook of gastrointestinal radiology. 2nd ed. Philadelphia, PA: Saunders, 2000:509-13.

16. Maglinte DD, Schultheis TE, Krol KL, Caudill LD Chernish SM, McCune WM. Survey of the esophagus during the upper gastrointestinal examination in 500 patientes. Radiology 1983;147:65-70.

17. Levine MS. Stomach and duodenum: differential diagnosis. In: Gore RM, Levine MS, eds. Textbook of gastrointestinal radiology. 2nd ed. Philadelphia, PA: Saunders, 2000:698-703. 